\title{
DSM-5: ¿Cambios significativos?
}

DSM-5: Significant changes?

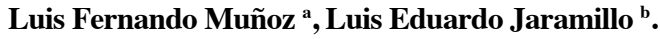

${ }^{a}$ Psiquiatra. ${ }^{b}$ Profesor departamento de Psiquiatría ${ }^{a y b}$ Universidad Nacional de Colombia.

Correspondencia: Luis Fernando Muñoz(lfmunozc@unal.edu.co)

Recibido: 10/04/2014; aceptado con modificaciones: 01/10/2014

RESUMEN: El DSM-5 salió a la luz en mayo de 2013, generando gran expectativa y controversia por sus cambios e inclusiones, como la eliminación de los ejes diagnósticos, la organización del manual en un modelo de "ciclo vital", la aparición de nuevas entidades y el ajuste en los criterios de diagnóstico de muchos trastornos. El objetivo del presente artículo es presentar una aproximación al manual desde una perspectiva latinoamericana, exponiendo las opiniones personales de los autores respecto a los principales cambios. PALABRAS CLAVE: Manual Diagnóstico y Estadístico de los Trastornos Mentales, Psicopatología, Revisión, Clasificación, Psiquiatría.
ABSTRACT: Since that DSM appeared on may of 2013, was generated an important expectative and controversy because of its changes in inclusions, the elimination of diagnostic axes, the manual's organization in a model of "life cycle", the emergence of new entities and the adjust in the criteria of diagnosis of many disorders. The aim of this paper is show an approach to manual from a Latin American perspective, and exposes the opinions of the authors regarding the major changes.

KEY WORDS: Diagnostic and Statistical Manual of Mental Disorders, Psychopathology, Review, Classification, Psychiatry.

Agradecimiento:

Al Dr. Mariano Hernández Monsalve, por leer el texto y aportar sugerencias que nos resultaron de gran utilidad.

\section{Introducción:}

El 18 de mayo de 2013, la Asociación Psiquiátrica Americana publicó el Manual de Diagnóstico Psiquiátrico (DSM-5), el cual fue el resultado de más de una década de investigación, con 13 grupos de trabajo, 6 grupos de estudio y más de 500 profesionales participando en cada uno de los mismos (1). Según el presidente del Task Force, David Kupfer, el DSM-5 fue diseñado pensando en facilitar la actualización del conocimiento relevante que se acumula en neurociencia, ciencias cognitivas, genética, y en la práctica clínica (2).

Es importante reconocer, que la clasificación previa de los diagnósticos psiquiátricos (DSM-IVR) tenía una serie de limitaciones, por eso era necesario buscar una mejor nosología psiquiátrica. Las limitaciones más notables del DSM-IVR eran entre otras: Altas tasas de comorbilidad entre los diagnósticos, un uso masivo y extendido de los diagnósticos "no especificados" y una creciente incapacidad para integrar los diagnósticos del DSM con los hallazgos de la investigación genética y neurobiológica (3-4). 
Además, los límites para las patologías que tratamos en psiquiatría se pueden ampliar fácilmente porque no hay una línea clara que separe las personas que están simplemente preocupadas, de aquellas con trastornos mentales leves (5). De hecho, los manuales diagnósticos en psiquiatría aparecieron en respuesta a la incertidumbre generada décadas atrás en el ámbito médico respecto a la validez de los diagnósticos psiquiátricos. Uno de los estudios más controversiales en ese sentido fue el de Rosenhan (6).

El objetivo del presente artículo es presentar una aproximación al manual desde una perspectiva latinoamericana, exponiendo las opiniones personales de los autores respecto a los principales cambios.

\section{Principales cambios:}

Un primer cambio es la desaparición de los ejes diagnósticos. Los autores han sustentado el cambio argumentando que se debió en gran parte a su incompatibilidad con los sistemas de diagnóstico en el resto de la medicina, así como el resultado de una decisión de colocar los trastornos de la personalidad y la discapacidad intelectual al mismo nivel que los otros trastornos mentales (7). Si bien por un lado tener un sistema multiaxial nos diferencia aún más del resto de las especialidades médicoquirúrgicas, que suelen ignorar dicha clasificación y enfocarse en el eje I únicamente; por otro lado, ha permitido que los pacientes sean considerados de manera integral y única cuando se realiza una valoración psiquiátrica. Además, para quienes el sistema multiaxial es importante, los autores aportan soluciones como emplear un diagnóstico de código $\mathrm{V}$ correspondiente (o en la lista del CIE-10 de código Z) en lugar del eje IV y en vez del puntaje GAF del eje $\mathrm{V}$, se puede emplear la escala de discapacidad de la OMS (WHODAS 2.0), que se encuentra en la sección III del DSM-5 (8). Esta última escala parece un poco más cercana a la realidad que el puntaje GAF del DSMIVr que implicaba una mayor subjetividad por las descripciones de los requisitos necesarios para obtener cada puntaje.

El DSM-5 está configurado en 3 secciones. El orden de los capítulos en la Sección II refleja un enfoque de "ciclo vital", con los trastornos más frecuentemente diagnosticados en la infancia al comienzo del manual, el primer capítulo es sobre los trastornos del neurodesarrollo, y al final del manual se encuentran los trastornos más frecuentes en las personas de edad avanzada, tales como los trastornos neurocognitivos (1). Consideramos que este diseño es más organizado con respecto al anterior y permite una lectura más estructurada.

De los cambios presentados en la sección II destacamos los siguientes:

- Cambios en terminología con mismos significados: Es de destacar el cambio de nombre de algunas categorías diagnósticas, permaneciendo, en esencia, con 
los mismos significados, y quizá con el fin de disminuir el estigma asociado con los trastornos mentales. Parte de eliminar el estigma, se trata de elegir las palabras adecuadas. Dentro de estos cambios, destacamos la Discapacidad intelectual (antiguo Retardo mental), la Disforia de género (antiguo Trastorno de identidad de género), el Trastorno por síntomas somáticos (que ahora abarca el término de Hipocondriasis, entre otros) y los Trastornos parafílicos (que reemplaza las Parafilias, comportamientos sexuales inusuales de muchas personas, no necesariamente patológicos).

- Incorporación de nuevos trastornos: El DSM-5 no es ajeno a esta polémica. Ya sucedió con el DSM-IV, manual para el cual de 94 nuevos diagnósticos sugeridos, el grupo de trabajo finalmente agregó sólo 2, pero esto no impidió la aparición inesperada de 3 modas diagnósticas dirigidas por el mercado (9). El DSM-5 incorporó nuevos diagnósticos como el Trastorno por atracones, cuya amplia e incluyente definición parece que aumentará el número de consultas y derivaciones de personas que comen en exceso, se cree que incluirá un $6 \%$ de la población general (10). Asimismo, el nuevo Trastorno de desregulación destructiva del estado de ánimo, cuya finalidad es reducir el diagnóstico de trastorno bipolar en la infancia, probablemente conducirá al etiquetamiento de los niños con otra entidad nosológica, pasando simplemente de tener un diagnóstico a otro (11-12), y cuyo llamativo nombre no deja de ser polémico. Además, el paso del apéndice B al cuerpo principal del DSM-5 del Trastorno disfórico premenstrual también genera controversia, por el posible manejo farmacológico que implica un diagnóstico nuevo, explicado probablemente por cambios hormonales fisiológicamente normales. Sin embargo, estudios epidemiológicos y clínicos han mostrado consistentemente que algunas mujeres experimentan un patrón de síntomas desagradables que inician en la fase lútea del ciclo menstrual y terminan rápidamente después del inicio de la menstruación, con prevalencias medias del 5\% en la población general (13). De otro lado, la aparición de una entidad nosológica definida como el Trastorno Neurocognitivo menor pretende detectar e intervenir precozmente para prevenir o retardar la progresión de una demencia incipiente. Para protegerse contra falsos positivos hay un criterio que requiere una evaluación cognitiva objetiva para confirmar que el individuo ha disminuido su desempeño cognitivo; sin embargo, se cree que incluirá alrededor del 13,5\% de la población general (10).

Por tanto, es inevitable prever que ante estos nuevos diagnósticos muy probablemente aparecerán demasiados nuevos "pacientes", que llevarán al uso masivo de fármacos.

- Los Trastornos psicóticos: Aquí destacamos que en la Esquizofrenia se hayan eliminado los subtipos y ese trato "especial" que recibían los síntomas de primer rango de Schneider. El impacto de este cambio en la práctica clínica será limitado 
debido a que menos del 2\% de la esquizofrenia del DSM-IV cumplía el criterio A exclusivamente en virtud de un solo síntoma de primer orden (14), mientras que la eliminación de los subtipos de la esquizofrenia representa una desviación importante de una tradición de cien años. Esto último, tendrá un impacto clínico relativamente pequeño excepto por los subtipos paranoide e indiferenciada, ya que los otros subtipos rara vez se utilizan en los sistemas de salud mental más comunes en todo el mundo (15) y se considera que tienen una estabilidad diagnóstica limitada, baja confiabilidad y pobre validez (16-17). Además, consideramos de relevancia pronóstica, evaluar la gravedad de los síntomas psicóticos empleando el enfoque dimensional propuesto en la sección III del manual, lo cual permite destacar la heterogeneidad que muestran en la práctica clínica los pacientes con respecto al tipo de síntomas y la gravedad expresada, mediante la evaluación de las siguientes dimensiones sintomáticas: positivos, negativos, afecto, desorganización, motor y cognitivo. De otro lado, creemos que se debe considerar con cautela el diagnóstico del Síndrome de Psicosis Atenuada, incluido en la Sección III del manual por su situación nosológica incierta, que al parecer presenta una alta tasa de falsos positivos en estudios realizados hasta la fecha (18) con el consabido riesgo del etiquetamiento y el uso inapropiado de antipsicóticos, especialmente en adolescentes y adultos jóvenes.

- Se hace una división de la sección de Trastornos afectivos en dos unidades, una en Espectro bipolar y trastornos relacionados y la otra en Trastornos depresivos. Este cambio nos parece que resalta la importancia de separar estas entidades en categorías diferentes, reconociendo la heterogeneidad que implicaba el término "Trastorno del estado de ánimo". Además, se añadió un especificador de "síntomas de ansiedad" tanto para las secciones Bipolar y Depresiva, lo cual debería fomentar la atención que merece este dominio de síntomas y reconocer que esta comorbilidad es bastante frecuente.

- En la categoría de los Trastornos depresivos, el duelo no excluye el diagnóstico de depresión. Este es quizás uno de los cambios más polémicos. Si bien los autores han esgrimido múltiples razones para omitir dicha exclusión, consideramos que la nota aclaratoria que intenta resolver esta difícil distinción entre duelo y depresión mayor puede resultar insuficiente para el clínico en formación y podrá llevar a la medicalización innecesaria de una de las experiencias más comúnmente vividas por todos los seres humanos, especialmente dado que hay mucha variabilidad individual y cultural en su afrontamiento (19-20). Quizá si queremos eliminar el estigma y los prejuicios que hay alrededor de la patología psiquiátrica, incluso desde otras ramas de la medicina, es importante evitar patologizar situaciones normales.

- El espectro Obsesivo Compulsivo, una nueva categoría diagnóstica, consideramos que reconoce la creciente evidencia que estos trastornos están relacio- 
nados entre sí en términos de una serie de validadores de diagnóstico (21). De hecho, al ser incluido como un subtipo de trastorno de ansiedad, el Trastorno Obsesivo Compulsivo muchas veces veía reducida su complejidad a la aparición o no de síntomas ansiosos, desconociendo sus demás diferencias con los otros trastornos de este grupo. Además, los especificadores de "insight" para el TOC creemos que han quedado redefinidos de una manera más clara en términos diagnósticos y terapéuticos.

- Los Trastornos somatomorfos sufrieron múltiples modificaciones: En primer lugar, su nombre cambia por Trastornos por síntomas somáticos, al parecer por el interés de evitar términos peyorativos y que refuercen el dualismo cartesiano “mente/cuerpo". Además, dado que en el DSM-IV, se produjo un solapamiento significativo entre todos los trastornos somatomorfos y una falta de claridad acerca de sus límites (22-23), se han eliminado los diagnósticos de Trastorno de somatización, Hipocondriasis, Trastorno por dolor y el Trastorno somatomorfo indiferenciado. Consideramos que este cambio si bien hace una nosología más simplificada, puede etiquetar como enfermos mentales a muchos individuos con enfermedades médicas que manifiesten una preocupación y sufrimiento más allá de lo esperable, que en sistemas de salud como el nuestro puede ser simplemente la consecuencia de una falta de atención oportuna. Es evidente que se requerirá formación clínica, experiencia y un juicio basado en las orientaciones como las contenidas en el DSM-5 para reconocer cuando los pensamientos, sentimientos y conductas del paciente son indicativos de un trastorno mental que pueda beneficiarse de un tratamiento enfocado (24).

- Los Trastornos de personalidad mantienen los mismos subtipos y clusters en la sección I. Sin embargo, la sección III incluye un enfoque alternativo para el diagnóstico de trastornos de la personalidad que se llama Modelo dimensional categórico híbrido alternativo. Este modelo mixto posee una serie de niveles jerárquicos distintos que permite un diagnóstico categorial y una aproximación al detalle dimensional sirviéndose de un conjunto de procedimientos estadísticos que se conocen como análisis factoriales (25-26). Este modelo dimensional de la personalidad fracasó cuando fue probado en pacientes en los ensayos de campo del proyecto de criterios DSM entre 2010 y 2012: Muchos psiquiatras que los emplearon llegaron a conclusiones diferentes (27). Las controvertidas dimensiones de los trastornos de personalidad, fueron votadas en contra por la Mesa Directiva de la APA en la reunión de planificación final en diciembre de 2012. Sin embargo, es esperable que en las versiones posteriores del manual, este modelo híbrido sea trasladado a la sección I, una vez los psiquiatras se familiaricen más con su uso y hayan más estudios que soporten su empleo.

- La Formulación cultural está incluida en la sección III del manual y es quizá una de las secciones que puede tener mayor aplicabilidad para la psiquiatría 
latinoamericana. Quizá lo más novedoso es la introducción de la guía de Entrevista de Formulación Cultural que fue probada en estudios de campo para uso clínico, la cual consta de 16 preguntas que se enfocan en la experiencia individual y los contextos sociales del problema clínico, estableciendo 4 dominios de evaluación: Definición cultural del problema, Percepciones culturales de la causa, contexto y apoyo, Factores culturales que afectan el auto-afrontamiento y la búsqueda de ayuda previa y Factores culturales que afectan la actual búsqueda de ayuda, incluyendo además una versión para informantes, con el fin de recolectar información colateral relevante de miembros de la familia y cuidadores (1). Consideramos que esto sin duda representa un avance con respecto al DSM-IV, donde la formulación cultural era muy precaria y no había una guía clara que permitiera evaluar mejor los aspectos culturales relevantes de cada paciente. Es de resaltar que la práctica psiquiátrica común suele dirigirse hacia la percepción por parte del psiquiatra acerca del problema del paciente, sin tomar en cuenta en muchas ocasiones la opinión de éste o su familia, por lo que es refrescante ver preguntas en dicha formulación enfocadas a evaluar la percepción propia del paciente de sus problemas, así como una evaluación detallada de los mecanismos de afrontamiento lo cual puede derivar en mejoras en la atención y tratamiento al obtener un mayor conocimiento del paciente, sin centrarse únicamente en los hallazgos psicopatológicos. Además, en este punto vale la pena mencionar el aporte a la llamada etnopsiquiatría de la Guía Latinoamericana de Diagnóstico Psiquiátrico - Versión Revisada (GLADP-VR) del año 2012 que especifica, de manera clara y concisa, unos síndromes culturales específicos y no específicos de América Latina de manera detallada (28).

\section{Discusión:}

La publicación de este nuevo manual Diagnóstico produce reacciones paradójicas. Por una parte parece acercarse más a la realidad de la práctica clínica simplificando varios elementos y haciendo una estructura más cercana al ciclo vital, por otra, da la sensación simplemente de haber hecho un ejercicio de reorganización mecánica, sin que ello implicara cambios profundos. Probablemente esto no se logra, porque al contrario de lo que a veces se publicita, no hemos logrado avanzar de manera significativa en los procesos fisiopatológicos y cognitivos subyacentes a los trastornos mentales. Queda entre el tintero también la pregunta de hacer un manual que contiene más de 300 diagnósticos, cuando en la vida real el porcentaje de los diagnósticos que hacemos se pueden resumir en no más de 10 categorías. ¿Valdría la pena ver el manual organizado no de acuerdo al ciclo vital, sino en relación con las 
ORIGINALES Y REVISIONES

frecuencias de las patologías (de mayor a menor frecuencia), lo cual permitiría hacer una búsqueda más fácil para la persona que consulta el manual?

En este artículo solo hemos mencionado los cambios más relevantes en nuestra perspectiva. Desde que se anunció su publicación para mediados de mayo de 2013, empezaron a plantearse una serie de polémicas acerca de los cambios anunciados. Por ejemplo, el Comité Internacional de respuesta al DSM-5, publicó una declaración de preocupación (29), en la cual hace un llamado a médicos, científicos, consumidores y otras partes interesadas, para evitar el uso del DSM-5 siempre que sea posible, y buscar activamente otras técnicas diagnósticas alternativas. Además, el 29 de abril de 2013, Thomas Insel, director del National Institute of Mental Health (NIMH), escribió en su blog que " "los pacientes con trastornos mentales se merecen algo mejor" (30). Insel señaló que a diferencia de los criterios de diagnóstico para otras enfermedades, los criterios del DSM-5 se basan en el consenso en lugar de medidas objetivas de laboratorio, careciendo de validez y explicó que el NIMH "reorientará su investigación fuera de las categorías del DSM-5". Para ello ha puesto en marcha el proyecto Research Domain Criteria $(\mathrm{RDoC})$ que pretende recopilar información procedente de la genética, las pruebas de neuroimagen, las ciencias cognitivas y otros datos fisiológicos, para identificar e integrar los componentes vinculados a los síntomas de enfermedad mental (31). De hecho, investigadores financiados en parte por el NIMH han encontrado que las cinco principales enfermedades mentales tienen variaciones genéticas comunes y la coincidencia era más alta entre la esquizofrenia y el trastorno bipolar, moderada para el trastorno bipolar y la depresión y el TDAH y la depresión, y baja entre la esquizofrenia y el autismo (32).

Quizá cabe plantearse si el modelo de clasificación que empleamos en psiquiatría carece de validez y el modelo a imitar ya no deba ser el Freudiano o el Kraepelinano, sino la revolución genética que tiene lugar en oncología, donde los investigadores y los médicos están empezando a clasificar y tratar el cáncer sobre la base del perfil genético detallado de un tumor en lugar de la parte del cuerpo en la que crece (33). De esta manera, han aparecido investigadores como Craddock y su colega Owen que han propuesto el espectro más radical de tipo dimensional hasta el momento (34), en el que cinco clases de trastornos mentales están dispuestos en un solo eje: Retraso mental, autismo, esquizofrenia, trastorno esquizoafectivo, trastorno bipolar / trastorno del estado de ánimo unipolar. Además, uno de los proyectos financiados por el NIMH, está examinando la anhedonia, como una condición que se encuentra en muchas enfermedades mentales, como la depresión y la esquizofrenia. Este grupo está estudiando la idea de que circuitos cerebrales disfuncionales desencadenan la liberación de citocinas inflamatorias y que estos direccionan la anhedonia suprimiendo la motivación y el placer. Los científicos planean investigar estos vínculos mediante análisis de expresión génica y neuroimágenes. En teoría, si se pudieron identificar este $\mathrm{u}$ otros mecanismos de la anhedonia, los pacientes podrían ser probados por ellos y tratados, independiente si tienen un diagnóstico DSM o no (35). 


\section{Conclusiones:}

- Es una clasificación congruente con las demás clasificaciones de la OMS, teniendo en cuenta que los psiquiatras somos los únicos que diagnosticábamos con un modelo multiaxial. Sin embargo, dicho enfoque diagnóstico permitió que los pacientes fueran considerados de manera integral y única cuando se realiza una valoración psiquiátrica.

- $\quad$ Seguimos sin tener marcadores biológicos con suficientes datos empíricos para hacer una nosología más clara. Si bien es cierto que los manuales DSM han sido diseñados para crear un lenguaje unificado para la investigación, siguen teniendo limitaciones en aplicar a los pacientes de la vida real muchos de los diagnósticos existentes en el manual, que quizá no responden a una lista de chequeo pero tienen condiciones que ameritan atención e intervención.

- Los nuevos diagnósticos y algunas de las modificaciones que se han introducido pueden producir una patologización y psiquiatrización de la vida cotidiana que incrementará de forma innecesaria, pero no inocua, el número de personas atendidas y medicalizadas por los profesionales de la salud mental.

- Mejor caracterización de algunas patologías: La eliminación de los subtipos de esquizofrenia, la división de los trastornos afectivos, la creación del espectro obsesivo-compulsivo y la formulación cultural, entre otros.

- Modelo sujeto a cambios - "Documento viviente": Uno de los aspectos más importantes del manual es que estará continuamente en revisión y sus autores aseguran que, de acuerdo a la evidencia que vaya surgiendo, irán apareciendo nuevas versiones, lo cual augura que no pasarán trece años hasta la siguiente actualización, como sucedió entre el DSM-IVr y el DSM-5. Sin embargo, este punto también debe ser considerado como una prueba más de la falta de fiabilidad y validez de las clasificaciones psiquiátricas y posiblemente mucho más de la del DSM-5 que generará muchos "falsos positivos" que serán sometidos a tratamientos innecesarios y iatrogénicos, cuyos efectos no serán revertidos con eventuales actualizaciones de la clasificación.

- Manual más organizado en el contexto de la edad - es decir, presentación de ciclo de vida del desarrollo dentro de cada capítulo - así como teniendo en cuenta el género y las diferencias culturales: ¿Valdría la pena ver el manual organizado por frecuencia de las patologías y no por ciclo vital?

- Los autores han reconocido públicamente sus falencias (36).

- 10 años de discusiones, grupos de trabajo, etc.: Quizá los pacientes, como lo afirma Insel, merecían algo mejor.

- $\quad$ Es una inversión de 25 millones de dólares (37).

- $\quad$ Puede ser confuso para aquellos que no sean del campo de la salud mental: Si bien el DSM-5 describe múltiples patologías de manera detallada, es un ma- 
nual que para personas que no son del campo de la salud mental o que están relacionadas de manera tangencial, les puede resultar en excesiva información. Incluso, a veces para el propio psiquiatra puede estar muy cargado en su contenido. ¿Valdría la pena un manual que contemplara únicamente las patologías más frecuentes y que tuviera unos criterios más sencillos?

- La validación de las entidades psiquiátricas desde el modelo propuesto por Robins y Guze (38) sigue siendo muy problemática.

- Pequeños cambios pueden tener grandes repercusiones clínicas, debido a la inclusión de nuevos diagnósticos y los ajustes que sufrieron otros como la Depresión mayor y los Trastornos somatomorfos.

Estamos ante una publicación que marcará el lenguaje psiquiátrico en los años venideros. Es obvio que como psiquiatras necesitamos estar familiarizados con los cambios presentados. Si bien el DSM-5 nos sigue dando un lenguaje común para hablar entre clínicos e investigadores, el diagnóstico de los trastornos mentales va más allá de una simple lista de chequeo de los criterios planteados por el manual y requiere un ejercicio mental mucho más complejo para poder entender lo que le pasa a nuestros pacientes.

\section{BIBLIOGRAFÍA:}

(1) American Psychiatric Association. Diagnostic and Statistical Manual of Mental Disorders, Fifth Edition. Washington, DC: American Psychiatric Association; 2013.

(2) Kupfer DJ, Kuhl EA, Regier DA. DSM-5-The Future Arrived. JAMA. 2013; 303(19):1974-1975.

(3) Frances A. The New Crisis in Confidence in Psychiatric Diagnosis. Ann Intern Med. 2013 Aug 6;159(3):221-2.

(4) Frances A. The past, present and future of psychiatric diagnosis. World Psychiatry 12.2:111-112. 2013.

(5) Batstra L, Frances A. Diagnostic inflation: Causes and a suggested cure. J Nerv Ment Dis. 2012 Jun;200(6):474-9.

(6) Rosenhan DL. On being sane in insane places. Science. 1973;179: 250-8.

(7) Kupfer DJ, Regier DA. Why all of medicine should care about DSM-5. JAMA. 2010; 303(19):1974-1975.

(8) Black D. Changes to DSM-5 are not as drastic as they may seem. Annals of Clinical Psychiatry. 2013;25(3):161.

(9) Batstra L, Hadders-Algra M, Nieweg E, Van Tol D, Pijl SJ, Frances A. Childhood emotional and behavioral problems: reducing overdiagnosis without risking undertreatment. Dev Med Child Neurol. 2012;54:492-4.

(10) Frances A.Opening Pandora's box: The 19 worst suggestions for DSM5. Psychiatric Times. 2010; 27: 9 . 
(11) Axelson D. Taking disruptive mood dysregulation disorder out for a test drive. Am J Psychiatry. 2013;170:136-139.

(12) Axelson D, Findling RL, Fristad MA, et al. Examining the proposed disruptive mood dysregulation disorder diagnosis in children in the Longitudinal Assessment of Manic Symptoms study. J Clin Psychiatry. 2012;73:1342-50.

(13) Epperson C, Steiner M, Hartlage A, et al. Premenstrual Dysphoric Disorder: Evidence for a New Category for DSM-5. Am J Psychiatry. 2012 May ; 169(5): 465-475.

(14) Tandon, R., Gaebel W., Barch D., et al. Definition and description of schizophrenia in the DSM-5, Schizophr Res. 2013 Oct;150(1):3-10.

(15) Keshavan, M.S., Nosology of psychoses in DSM5: Inches ahead but miles to go, Schizophr Res. 2013 Oct;150(1):40-1.

(16) Tandon R. Schizophrenia and other Psychotic Disorders in DSM-5: Clinical Implications of Revisions from DSM-IV. Clin Schizophr Relat Psychoses. 2013 Apr;7(1):16-9.

(17) Keshavan, M.S., Clementz, B.A., Pearlson, G.D., Sweeney, J.A., Tamminga, C.A., 2013. Reimagining psychoses: an agnostic approach to diagnosis. Schizophr. Res. 146 (1-3), 10-16.

(18) Tsuang M., Van Os J., Tandon R., Barch DM., Bustillo J., Gaebel W., et al. Attenuated psychosis syndrome in DSM-5. Schizophr Res. 2013 Oct;150(1):31-5.

(19) Wakefield JC, First MD. Validity of the bereavement exclusion to major depression: does the empirical evidence support the proposal to eliminate the exclusion in DSM-5? World Psychiatry. 2012;11:3-10.

(20) Pies R. After bereavement, is it "normal grief" or major depression? Psychiatric Times (revista electrónica) 2012: Disponible en www.psychiatrictimes.com/mdd/content/ article/10168/2035804

(21) Brakoulias V. Diagnostic subtyping of obsessive-compulsive disorder: Have we got it all wrong? Aust N Z J Psychiatry 2013 47: 23.

(22) Dimsdale JE. Medically unexplained symptoms: a treacherous foundation for somatoform disorders? Psychiatr Clin North Am 2011;34:511-3.

(23) Creed F, Gureje O. Emerging themes in the revision of the classification of somatoform disorders. Int Rev Psychiatry 2012;24:556-67.

(24) Frances A. The new somatic symptom disorder in DSM-5 risks mislabeling many people as mentally ill. BMJ 2013;346:f1580.

(25) López-Santín J., Molins F., Litvan L. Trastornos de personalidad en el DSM-5. Una aproximación crítica. Rev. Asoc. Esp. Neuropsiq., 2013; 33 (119) 497-510.

(26) Trull TJ, Tragesser SL, Solhan M, Schwartz-Mette R: Dimensional models of personality disorder: Diagnostic and Statistical Manual of Mental Disorders Fifth Edition and beyond. Curr Opin Psychiatry. 2007 Jan;20(1):52-6.

(27) Adam D. Mental health: On the spectrum. Nature. 2013 Apr 25;496(7446):416-8.

(28) Asociación Psiquiátrica de América Latina: Sección de Diagnóstico y Clasificación. Guía Latinoamericana de Diagnóstico Psiquiátrico - Versión Revisada (GLADP-VR). América Latina 2012.

(29) Comité Internacional de Respuesta al DSM-5. DECLARACIÓN PÚBLICA DE PREOCUPACIÓN POR LA PRÓXIMA PUBLICACIÓN DEL DSM-5. Disponible online en $w w w$. infocoponline.es/pdf/Declaración.pdf 
(30) Insel $\mathrm{T}$ (2013). Disponible en http://www.nimh.nih.gov/about/director/2013/ transforming-diagnosis.shtml

(31) Insel, T., Cuthbert, B., Garvey, M., et al., 2010. Research Domain Criteria (RDoC): toward a new classification framework in mental disorders. Am J Psychiatry. 167; 748-751.

(32) Cross-Disorder Group of the Psychiatric Genomics Consortium. Genetic relationship between five psychiatric disorders estimated from genome-wide SNPs. Nat Genet. 2013 Sep;45(9):98494.

(33) Malhi G. DSM-5: Il buono, il cattivo, il brutto. Aust N Z J Psychiatry 2013 47: 595.

(34) Craddock N, Owen MJ. The Kraepelinian dichotomy - going, going... but still not gone. Br J Psychiatry. 2010 Feb;196(2):92-5.

(35) Savitz J, Frank MB, Victor T, et al. Inflammation and neurological disease-related genes are differentially expressed in depressed patients with mood disorders and correlate with morphometric and functional imaging abnormalities. Brain Behav Immun. 2013 Jul;31:161-71.

(36) Kupfer DJ, Regier DA. Neuroscience, clinical evidence, and the future of psychiatric classification in DSM-5. Am. J. Psychiatry. 2011 Jul;168(7):672-4.

(37) Sachdev P. Is DSM-5 defensible? Aust N Z J Psychiatry 2013 47: 10.

(38) Feighner J., Robins E., Guze S. Diagnostic Criteria for Use in Psychiatric Research. Arch Gen Psychiatry. 1972;26(1):57-63. 